Document downloaded from:

http://hdl.handle.net/10251/52821

This paper must be cited as:

Benítez López, J.; Liu, X.; Zhu, T. (2011). Additive results for the group inverse in an algebra with applications to block operators. Linear and Multilinear Algebra. 59(3):279-289. doi:10.1080/03081080903410262.

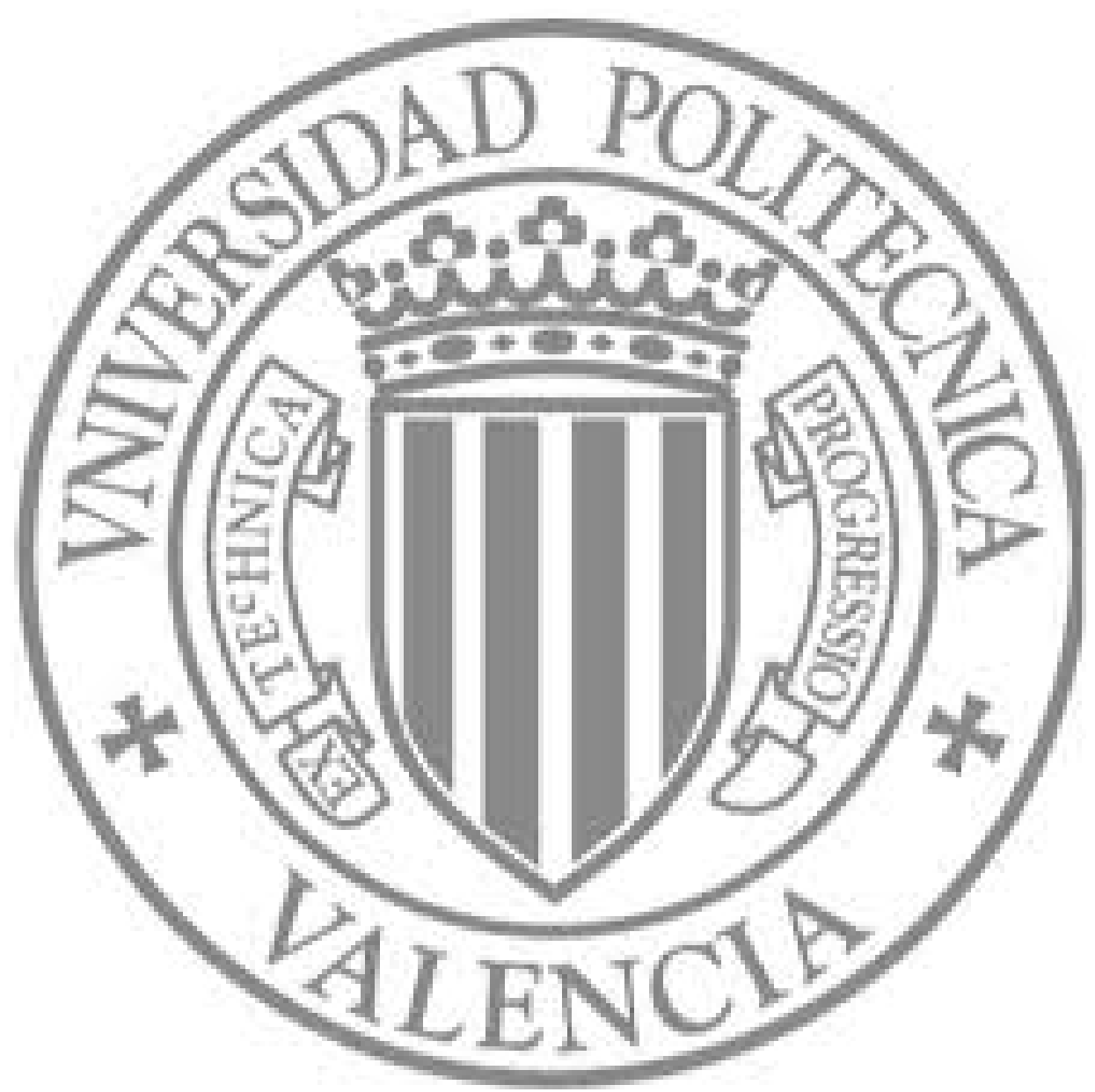

The final publication is available at

http://dx.doi.org/10.1080/03081080903410262

Copyright Taylor \& Francis 


\title{
Additive results for the group inverse in an algebra with applications to block operators *
}

\author{
Julio Benítez ${ }^{\text {a }} \quad$ Xiaoji Liu ${ }^{\text {b }} \quad$ Tongping $Z \mathrm{Zu}^{\mathrm{c}}$ \\ a Departamento de Matemática Aplicada, Instituto de Matemática Multidisciplinar, \\ Universidad Politécnica de Valencia, Camino de Vera s/n, 46022, Valencia, Spain. \\ b, c College of Mathematics and Computer Science, \\ Guangxi University for Nationalities Nanning 530006, P.R. of China
}

\begin{abstract}
We derive a very short expression for the group inverse of $a_{1}+\cdots+a_{n}$ when $a_{1}, \ldots, a_{n}$ are elements in an algebra having group inverse and satisfying $a_{i} a_{j}=0$ for $i<j$. We apply this formula in order to find the group inverse of $2 \times 2$ block operators under some conditions.
\end{abstract}

AMS classification: $15 \mathrm{~A} 09 ; 47 \mathrm{~A} 05$

Key words: algebra, group inverse, block operators.

\section{Introduction}

Throughout this paper, $\mathcal{A}$ will denote an algebra with unity 1 and we will denote by $\mathcal{A}^{-1}$ the subset of invertible elements of $\mathcal{A}$. An idempotent $p \in \mathcal{A}$ satisfies $p=p^{2}$. An element $a \in \mathcal{A}$ is said to have a group inverse if there exists $x \in \mathcal{A}$ such that

$$
a x a=a, \quad x a x=x, \quad a x=x a .
$$

It can easily be proved that if $a \in \mathcal{A}$ has a group inverse, then the element $x$ satisfying (1.1) is unique, and under this situation, we shall write $x=a^{\#}$. The subset of $\mathcal{A}$ consisting of elements of $\mathcal{A}$ that have a group inverse inverse will be denoted by $\mathcal{A}^{\#}$. For an arbitrary algebra $\mathcal{A}$, it is not true that $\mathcal{A}=\mathcal{A}^{\#}$. Even when $\mathcal{A}$ is the set of complex matrices of order $n \times n$ (denoted by $\mathbb{C}^{n \times n}$ ), the equality $\mathcal{A}=\mathcal{A}^{\#}$ does not hold (an equivalent condition for $A \in \mathbb{C}^{n \times n}$ having a group inverse is that $\operatorname{rank}(A)=\operatorname{rank}\left(A^{2}\right)$, see for example [1]).

The group inverse in a Banach algebra is a particular case of the Drazin inverse (see [12]). Since the group inverse of $a \in \mathcal{A}^{\#}$ must commute with $a$, the study of such kind on invertibility resembles to the study of the elements of a $C^{*}$-algebra that commute with their Moore-Penrose inverse (see [2, 12]). Following the proof of Theorem 2.1 in [2], we can establish the following result:

\footnotetext{
${ }^{*}$ The second and the third authors are supported by Guangxi Science Foundation (0640016, 0832084).

${ }^{\dagger}$ Email: jbenitez@mat.upv.es

${ }^{\ddagger}$ Email: xiaojiliu72@yahoo.com.cn
} 
Theorem 1.1. Let $\mathcal{A}$ be an algebra with unity 1 and $a \in \mathcal{A}$. Then the following conditions are equivalent:

(i) There exists a unique idempotent $p$ such that $a+p \in \mathcal{A}^{-1}$ and $a p=p a=0$.

(ii) $a \in \mathcal{A}^{\#}$.

Following [13], we denote by $a^{\pi}$ the unique idempotent satisfying condition (i) of Theorem 1.1 for a given $a \in \mathcal{A}^{\#}$. Let us remark that $a a^{\pi}=a^{\pi} a=0$ and from the proof of Theorem 1.1 we have

$$
a^{\pi}=1-a a^{\#}=1-a^{\#} a \quad \text { and } \quad a^{\#}=\left(a+a^{\pi}\right)^{-1}-a^{\pi} .
$$

The idempotent $a^{\pi}$ will be named the spectral idempotent of $a$ corresponding to 0 .

\section{Some additive results for the group inverse in a algebra}

Recently, there has been interest in giving formulae for the Drazin inverse of a sum of two matrices (or two operators in a Hilbert space) under some conditions, see $[11,10,7,8]$ and references therein. In [3] it was proved the following result (among others). If $T_{1}, T_{2} \in \mathbb{C}^{n \times n}$ are two $k$-potent matrices for some natural $k>1$ (a matrix $X$ is said to be $k$-potent when $\left.X^{k}=K\right)$ such that $T_{1} T_{2}=0$ and $c_{1}, c_{2}$ are two nonzero complex numbers, then $c_{1} T_{1}+c_{2} T_{2}$ is group invertible and

$$
\left(c_{1} T_{1}+c_{2} T_{2}\right)^{\#}=c_{1}^{-1}\left(I_{n}-T_{2}^{k-1}\right) T_{1}^{k-2}+c_{2}^{-1} T_{2}^{k-2}\left(I_{n}-T_{1}^{k-1}\right) .
$$

In order to prove (2.3) the authors used block matrices and spectral theory for diagonalizable matrices (which means that the setting of the proof was finite-dimensional).

Next result generalizes the expression (2.3) in a algebra setting without considering the dimension of the space under consideration. First of all, let us see how (2.3) can be generalized. Let us remark that if $T$ is a $k$-potent matrix for some natural $k>1$, then $T$ is group invertible and $T^{\#}=T^{k-2}$ as is easily seen from the definition of the group inverse (see equations (1.1)). Observe that under this situation one has from (1.2) that $T^{k-1}=T T^{k-2}=T T^{\#}=I_{n}-T^{\pi}$, where $I_{n}$ denotes the identity matrix of order $n$. Moreover, it is very simple to check that if $A$ is any group invertible matrix and $c$ is a nonzero complex number, then $(c A)^{\#}=c^{-1} A^{\#}$. Hence, the formula (2.3) can be written

$$
\left(c_{1} T_{1}+c_{2} T_{2}\right)^{\#}=T_{2}^{\pi}\left(c_{1} T_{1}\right)^{\#}+\left(c_{2} T_{2}\right)^{\#} T_{1}^{\pi} .
$$

A related result in the setting of operators in a Banach space and Drazin inverses was given in $[6]$.

Theorem 2.1. Let $\mathcal{A}$ be an algebra with unity. If $a, b \in \mathcal{A}^{\#}$ satisfy $a b=0$, then $a+b \in \mathcal{A}^{\#}$ and

$$
(a+b)^{\#}=b^{\pi} a^{\#}+b^{\#} a^{\pi} .
$$

Proof. Let us denote $x=b^{\pi} a^{\#}+b^{\#} a^{\pi}$. In order to prove the formula of the theorem, we will check $(a+b) x=x(a+b),(a+b) x(a+b)=a+b$, and $x(a+b) x=x$. Before doing this, let us observe that $a^{\#} b=0$ (since $\left.a^{\#} b=a^{\#} a a^{\#} b=\left(a^{\#}\right)^{2} a b=0\right), a^{\pi} b=b$ (since 
$\left.a^{\pi} b=\left(1-a a^{\#}\right) b=b\right), a b^{\#}=0$ (since $\left.a b^{\#}=a b^{\#} b b^{\#}=a b\left(b^{\#}\right)^{2}=0\right)$, and $a b^{\pi}=a$ (since $\left.a b^{\pi}=a\left(1-b b^{\#}\right)=a\right)$. Next we simplify $(a+b) x$ and $x(a+b)$ :

$$
(a+b) x=(a+b)\left(b^{\pi} a^{\#}+b^{\#} a^{\pi}\right)=a b^{\pi} a^{\#}+a b^{\#} a^{\pi}+b b^{\pi} a^{\#}+b b^{\#} a^{\pi}=a a^{\#}+b b^{\#} a^{\pi},
$$

and

$$
\begin{aligned}
& x(a+b)=\left(b^{\pi} a^{\#}+b^{\#} a^{\pi}\right)(a+b)=b^{\pi} a^{\#} a+b^{\pi} a^{\#} b+b^{\#} a^{\pi} a+b^{\#} a^{\pi} b \\
& \quad=b^{\pi} a^{\#} a+b^{\#} b=\left(1-b b^{\#}\right) a^{\#} a+b^{\#} b=a^{\#} a+b b^{\#}\left(1-a^{\#} a\right)=a^{\#} a+b b^{\#} a^{\pi} .
\end{aligned}
$$

This proves $(a+b) x=x(a+b)$. Now,

$$
(a+b) x(a+b)=\left(a^{\#} a+b b^{\#} a^{\pi}\right)(a+b)=a^{\#} a^{2}+a^{\#} a b+b b^{\#} a^{\pi} a+b b^{\#} a^{\pi} b=a+b
$$

and

$$
\begin{aligned}
& x(a+b) x=\left(b^{\pi} a^{\#}+b^{\#} a^{\pi}\right)\left(a^{\#} a+b b^{\#} a^{\pi}\right) \\
& \quad=b^{\pi}\left(a^{\#}\right)^{2} a+b^{\pi} a^{\#} b b^{\#} a^{\pi}+b^{\#} a^{\pi} a^{\#} a+b^{\#} a^{\pi} b b^{\#} a^{\pi}=b^{\pi} a^{\#}+b^{\#} a^{\pi} .
\end{aligned}
$$

The theorem is proved.

Corollary 2.1. Let $\mathcal{A}$ be an algebra with unity. If $a, b \in \mathcal{A}^{\#}$ satisfy $a b=0=b a$, then $a+b \in \mathcal{A}^{\#}$ and

$$
(a+b)^{\#}=a^{\#}+b^{\#} .
$$

Proof. By Theorem 2.1 we have $a+b \in \mathcal{A}^{\#}$ and $(a+b)^{\#}=b^{\pi} a^{\#}+b^{\#} a^{\pi}$. Let us simplify each summand under the condition $b a=0$. Let us remark that $b a^{\#}=b a^{\#} a a^{\#}=b a\left(a^{\#}\right)^{2}=0$ and similarly, one has $b^{\#} a=0$. Now, $b^{\pi} a^{\#}=\left(1-b^{\#} b\right) a^{\#}=a^{\#}$ and $b^{\#} a^{\pi}=b^{\#}\left(1-a a^{\#}\right)=b^{\#}$. Hence, the corollary is proved.

Theorem 2.2. Let $a_{1}, \ldots, a_{n}$ be elements in an algebra having group inverse. If $a_{i} a_{j}=0$, for $i<j$ and $i, j \in\{1,2, \ldots, n\}$, then $a_{1}+\cdots+a_{n} \in \mathcal{A}^{\#}$ and

$$
\left(a_{1}+\cdots+a_{n}\right)^{\#}=a_{n}^{\pi} \cdots a_{2}^{\pi} a_{1}^{\#}+a_{n}^{\pi} \cdots a_{3}^{\pi} a_{2}^{\#} a_{1}^{\pi}+\cdots+a_{n}^{\pi} a_{n-1}^{\#} a_{n-2}^{\pi} \cdots a_{1}^{\pi}+a_{n}^{\#} a_{n-1}^{\pi} \cdots a_{1}^{\pi} .
$$

Proof. This formula will be proved by induction on $n$. For $n=2$, the formula is simply Theorem 2.1. Assume that the theorem is true when the number of summands is less than $n$. By the induction hypothesis, we have that $a_{2}+\cdots+a_{n} \in \mathcal{A}^{\#}$ and

$$
\left(a_{2}+\cdots+a_{n}\right)^{\#}=a_{n}^{\pi} \cdots a_{3}^{\pi} a_{2}^{\#}+a_{n}^{\pi} \cdots a_{4}^{\pi} a_{3}^{\#} a_{2}^{\pi}+\cdots+a_{n}^{\pi} a_{n-1}^{\#} a_{n-2}^{\pi} \cdots a_{2}^{\pi}+a_{n}^{\#} a_{n-1}^{\pi} \cdots a_{2}^{\pi} .
$$

Since $a_{1}$ and $a_{2}+\cdots+a_{n}$ have group inverse and $a_{1}\left(a_{2}+\cdots+a_{n}\right)=0$, by Theorem 2.1, we get $a_{1}+a_{2}+\cdots+a_{n} \in \mathcal{A}^{\#}$ and

$$
\left(a_{1}+a_{2}+\cdots+a_{n}\right)^{\#}=\left(a_{2}+\cdots+a_{n}\right)^{\pi} a_{1}^{\#}+\left(a_{2}+\cdots+a_{n}\right)^{\#} a_{1}^{\pi} .
$$

Using (2.4) we get

$$
\left(a_{1}+a_{2}+\cdots+a_{n}\right)^{\#}=\left(a_{2}+\cdots+a_{n}\right)^{\pi} a_{1}^{\#}+a_{n}^{\pi} \cdots a_{3}^{\pi} a_{2}^{\#} a_{1}^{\pi}+\cdots+a_{n}^{\#} a_{n-1}^{\pi} \cdots a_{1}^{\pi} .
$$

Therefore, in order to finish the proof, it is enough to demonstrate

$$
\left(a_{2}+\cdots+a_{n}\right)^{\pi}=a_{n}^{\pi} \cdots a_{2}^{\pi} .
$$


We are going to prove (2.5) by induction on $n$. If $n=2$, there is nothing to prove. Assume that

$$
\left(a_{2}+\cdots+a_{n-1}\right)^{\pi}=a_{n-1}^{\pi} \cdots a_{2}^{\pi}
$$

holds. By the hypothesis of the Theorem, we easily get when $i<j$ and $i, j \in\{1, \cdots n\}$ that $a_{i} a_{j}^{\#}=0$ and $a_{i} a_{j}^{\pi}=a_{i}$. Let us denote

$$
x_{2}=a_{n}^{\pi} \cdots a_{3}^{\pi} a_{2}^{\#}, \quad \cdots, \quad x_{n-1}=a_{n}^{\pi} a_{n-1}^{\#} a_{n-2}^{\pi} \cdots a_{2}^{\pi}, \quad x_{n}=a_{n}^{\#} a_{n-1}^{\pi} \cdots a_{2}^{\pi}
$$

and

$$
y_{2}=a_{n-1}^{\pi} \cdots a_{3}^{\pi} a_{2}^{\#}, \quad \cdots, \quad y_{n-1}=a_{n-1}^{\#} a_{n-2}^{\pi} \cdots a_{2}^{\pi} .
$$

Observe that $a_{n}^{\pi} y_{i}=x_{i}$ holds for $i=2, \ldots, n-1$. Now, from $(2.4)$

$$
\begin{aligned}
\left(\sum_{i=2}^{n} a_{i}\right)^{\pi} & =1-\left(\sum_{i=2}^{n} a_{i}\right)\left(\sum_{i=2}^{n} a_{i}\right)^{\#} \\
& =1-\left(\sum_{i=2}^{n} a_{i}\right)\left(\sum_{i=2}^{n} x_{i}\right) \\
& =1-\left(a_{n}+\sum_{i=2}^{n-1} a_{i}\right)\left(x_{n}+\sum_{i=2}^{n-1} x_{i}\right) \\
& =1-a_{n} x_{n}-a_{n} \sum_{i=2}^{n-1} x_{i}-\sum_{i=2}^{n-1} a_{i} x_{n}-\left(\sum_{i=2}^{n-1} a_{i}\right)\left(\sum_{i=2}^{n-1} x_{i}\right) \\
& =1-a_{n} x_{n}-a_{n} \sum_{i=2}^{n-1} a_{n}^{\pi} y_{i}-\sum_{i=2}^{n-1} a_{i} x_{n}-\left(\sum_{i=2}^{n-1} a_{i}\right)\left(\sum_{i=2}^{n-1} a_{n}^{\pi} y_{i}\right) \\
& =1-\sum_{i=2}^{n} a_{i} x_{n}-\left(\sum_{i=2}^{n-1} a_{i}\right)\left(\sum_{i=2}^{n-1} y_{i}\right) .
\end{aligned}
$$

On the other hand, since (2.6) was assumed, we have

$$
1-\left(\sum_{i=2}^{n-1} a_{i}\right)\left(\sum_{i=2}^{n-1} a_{i}\right)^{\#}=a_{n-1}^{\pi} \cdots a_{2}^{\pi} .
$$

Since we assume that the formula stated in the theorem holds when the number of summands is less than $n$, we have

$$
\left(\sum_{i=2}^{n-1} a_{i}\right)^{\#}=\sum_{i=2}^{n-1} y_{i}
$$

hence

$$
1-\left(\sum_{i=2}^{n-1} a_{i}\right)\left(\sum_{i=2}^{n-1} y_{i}\right)=a_{n-1}^{\pi} \cdots a_{2}^{\pi} .
$$


Therefore, from (2.7) and (2.8) and recalling $a_{2} a_{n}^{\#}=\cdots=a_{n-1} a_{n}^{\#}=0$, we obtain

$$
\begin{aligned}
\left(\sum_{i=2}^{n} a_{i}\right)^{\pi} & =a_{n-1}^{\pi} \cdots a_{2}^{\pi}-\left(\sum_{i=2}^{n} a_{i}\right) x_{n} \\
& =a_{n-1}^{\pi} \cdots a_{2}^{\pi}-\left(\sum_{i=2}^{n} a_{i}\right) a_{n}^{\#} a_{n-1}^{\pi} \cdots a_{2}^{\pi} \\
& =a_{n-1}^{\pi} \cdots a_{2}^{\pi}-a_{n} a_{n}^{\#} a_{n-1}^{\pi} \cdots a_{2}^{\pi} \\
& =\left(1-a_{n} a_{n}^{\#}\right) a_{n-1}^{\pi} \cdots a_{2}^{\pi} \\
& =a_{n}^{\pi} a_{n-1}^{\pi} \cdots a_{2}^{\pi} .
\end{aligned}
$$

And the theorem is proved.

\section{Applications}

We will use Theorem 2.1 to find the group inverse of a block matrix

$$
M=\left[\begin{array}{cc}
A & B \\
C & D
\end{array}\right]
$$

under some conditions on blocks $A, B, C$, and $D$. The main idea is to decompose $M=$ $M_{1}+M_{2}$, where matrices $M_{1}$ and $M_{2}$ contain some blocks of the matrix $M$, and these matrices $M_{1}$ and $M_{2}$ satisfy conditions of Theorem 2.1, i.e., $M_{1}, M_{2}$ are group invertible and $M_{1} M_{2}=0$. A prior work (for the Drazin inverse) can be founded in [9].

But, instead of establishing the main results in the setting of matrix theory (recall that the algebra composed of complex $n \times n$ matrices has finite dimension), we will work in an arbitrary algebra. We believe that avoiding the use of the spatial arguments in the standard $n$ dimensional Euclidean space in favor of simpler algebraic techniques gives a greater insight into this problem. As simple consequences we obtain results on matrices and bounded operators on Banach spaces.

We generalize the use of block matrices to the setting of unital algebras in the following lines. For any idempotent $p \in \mathcal{A}$, we shall denote $\bar{p}=1-p$. Evidently we have that $\bar{p}$ is also an idempotent and $p \bar{p}=\bar{p} p=0$. Now, any $a \in \mathcal{A}$ has the following matrix representation (see [5, Lemma 2.1]):

$$
a \equiv\left[\begin{array}{cc}
\text { pap } & p a \bar{p} \\
\bar{p} a p & \bar{p} a \bar{p}
\end{array}\right]
$$

Also, recall that since $p$ is an idempotent, $p \mathcal{A} p$ and $\bar{p} \mathcal{A} \bar{p}$ are algebras with units $p$ and $\bar{p}$, respectively. Such representation is useful to deal with block matrices or operators in direct sums of Banach spaces.

1. Let $\mathbb{C}^{n \times m}$ denote the set composed of $n \times m$ complex matrices and $I_{n}$ the identity matrix of order $n$. If $P \in \mathbb{C}^{n \times n}$ is the idempotent given by $P=S\left(I_{r} \oplus 0\right) S^{-1}$, where $S \in \mathbb{C}^{n \times n}$ is nonsingular, then any $M \in \mathbb{C}^{n \times n}$ can be represented using (3.10). Let us write $M$ as

$$
M=S\left[\begin{array}{ll}
A & B \\
C & D
\end{array}\right] S^{-1}, \quad A \in \mathbb{C}^{r \times r}, D \in \mathbb{C}^{(n-r) \times(n-r)} .
$$


If we compute, for example, $P M P$ we have

$$
P M P=S\left[\begin{array}{cc}
I_{r} & 0 \\
0 & 0
\end{array}\right]\left[\begin{array}{cc}
A & B \\
C & D
\end{array}\right]\left[\begin{array}{cc}
I_{r} & 0 \\
0 & 0
\end{array}\right] S^{-1}=S\left[\begin{array}{cc}
A & 0 \\
0 & 0
\end{array}\right] S^{-1}
$$

Hence the block $A$ in (3.11) can be identified with $P M P$. Similarly, we can identify the remaining blocks in (3.11) with the different products $P M \bar{P}, \bar{P} M P$, and $\bar{P} M \bar{P}$.

2. If $\mathcal{X}$ and $\mathcal{Y}$ are two Banach spaces, let us denote $\mathcal{B}(\mathcal{X}, \mathcal{Y})$ the algebra composed of bounded operators from $X$ to $y$, and $\mathcal{B}(X)=\mathcal{B}(X, X)$. If $P: X \times y \rightarrow X \times y$ denotes the projection given by $P(x, y)=(x, 0)$, then any $M \in \mathcal{B}(X \times y)$, written as $M(x, y)=$ $\left(M_{1}(x, y), M_{2}(x, y)\right)$, can be represented using (3.10). For example, if we compute $P M P$ we have

$$
P M P(x, y)=P M(x, 0)=P\left(M_{1}(x, 0), M_{2}(x, 0)\right)=\left(M_{1}(x, 0), 0\right) .
$$

Thus, $P M P$ can be identified with the operator in $\mathcal{B}(\mathcal{X})$ given by $x \mapsto M_{1}(x, 0)$. Similarly, $P M \bar{P}$ can be identified with the operator in $\mathcal{B}(\mathcal{Y}, \mathcal{X})$ given by $y \mapsto M_{1}(0, y)$, and so on.

In next result, we describe the elements having an inverse or a group inverse in the subalgebra $p \mathcal{A} p$, where $p$ is a nontrivial idempotent of $\mathcal{A}$. It is interesting to observe that $p a p$ is not invertible in $\mathcal{A}$ for any $a \in \mathcal{A}$, in fact, if there exists $x \in \mathcal{A}$ such that papx $=1$, then premultiplying by $\bar{p}$ we get $0=\bar{p}$, which contradicts the non-triviality of $p$. Contrarily, as we will see, the group inverse behaves differently. We denote $\operatorname{inv}(a, \mathcal{B})$ and $\#(a, \mathcal{B})$ the inverse and the group inverse, respectively in the subalgebra $\mathcal{B} \subset \mathcal{A}$ of $b \in \mathcal{B}$, if exists such inverse.

Theorem 3.1. Let $\mathcal{A}$ be an algebra with unity, $p \in \mathcal{A}$ an idempotent, and $a \in \mathcal{A}$. Then

(i) $p a p \in(p \mathcal{A} p)^{-1}$ if and only if pap $+\bar{p} \in \mathcal{A}^{-1}$. Under this situation one has $\operatorname{inv}(p a p, p \mathcal{A} p)+$ $\bar{p}=(p a p+\bar{p})^{-1}$.

(ii) If pap $\in \mathcal{A}^{\#}$, then $(\text { pap })^{\#} \in p \mathcal{A} p$.

(iii) pap $\in(p \mathcal{A} p)^{\#}$ if and only if pap $\in \mathcal{A}^{\#}$. Under this situation one has $\#(p a p, p \mathcal{A} p)=$ $(\text { pap })^{\#}$.

Proof. (i) Assume that $p a p \in(p \mathcal{A} p)^{-1}$. Having in mind that $p$ is the unity in the subalgebra $p \mathcal{A} p$, there exists $b \in \mathcal{A}$ such that $(p a p)(p b p)=p$ and $(p b p)(p a p)=p$, i.e., $p a p b p=p$ and pbpap $=p$. Now we have

$$
(p a p+\bar{p})(p b p+\bar{p})=p a p b p+p a p \bar{p}+\bar{p} p b p+\bar{p}^{2}=p+\bar{p}=1,
$$

and similarly $(p b p+\bar{p})(p a p+\bar{p})=1$ holds. This proves $p a p+\bar{p} \in \mathcal{A}^{-1}$ and $(p a p+\bar{p})^{-1}=$ $p b p+\bar{p}=\operatorname{inv}(p a p, p \mathcal{A} p)+\bar{p}$.

Assume now that pap $+\bar{p} \in \mathcal{A}^{-1}$, or in other words, there exists $x \in \mathcal{A}$ such that (pap + $\bar{p}) x=1$ and $x($ pap $+\bar{p})=1$, i.e., pap $x+\bar{p} x=1$ and $x p a p+x \bar{p}=1$. We have

$$
(\text { pap })(\text { pxp })=(\text { papx }) p=(1-\bar{p} x) p=p-\bar{p} x p .
$$

Premultiplying by $p$ we get $(p a p)(p x p)=p$. Similarly we have $(p x p)(p a p)=p$. 
(ii) Since $p a p \in \mathcal{A}^{\#}$, there exists $x \in \mathcal{A}$ such that $x=(\text { pap })^{\#}$, i.e.,

$$
\text { papx }=\text { xрар, } \quad \text { рархрар }=\text { рар, } \quad \text { храрx }=x .
$$

We have to prove $x \in p \mathcal{A} p$. Since $\bar{p} x=\bar{p} x p a p x=\bar{p} p a p x^{2}=0$, we obtain $x=p x$. In a similar way, since $x \bar{p}=x p a p x \bar{p}=x^{2} p a p \bar{p}=0$, we get $x=x p$. Therefore, $x=p x p$ holds, and this item is proved.

(iii) It is evident from the definition of the group inverse that $p a p \in(p \mathcal{A} p)^{\#}$ implies pap $\in \mathcal{A}^{\#}$ and $\#($ pap,$p \mathcal{A} p)=(\text { pap })^{\#}$.

Assume that $p a p \in \mathcal{A}^{\#}$. There exists $b \in \mathcal{A}$ such that

$$
(\text { pap }) b=b(\text { pap }), \quad(\text { pap }) b(\text { pap })=p a p, \quad b(\text { pap }) b=b .
$$

Pre and postmultiplying the above equalities by $p$ and having in mind $p^{2}=p$ we have

$$
(p a p)(p b p)=(p b p)(p a p), \quad(p a p) b(p a p)=p a p, \quad p b(p a p) b p=p b p .
$$

These equalities mean $p a p \in(p \mathcal{A} p)^{\#}$. The proof is finished.

As we said before, one of our purposes is to find the group inverse of the $2 \times 2$ block matrix $M$ represented in (3.9) under some conditions on the sub-blocks of $M$. One idea is to decompose

$$
\left[\begin{array}{ll}
A & B \\
C & D
\end{array}\right]=\left[\begin{array}{cc}
A & B \\
0 & 0
\end{array}\right]+\left[\begin{array}{cc}
0 & 0 \\
C & D
\end{array}\right]
$$

and apply Theorem 2.1. Of course, we must know when is it possible to find the group inverse of each summand in the right-hand of the above expression. In 2001, Cao [4] gave the following result:

Theorem 3.2. If $A \in \mathbb{C}^{r \times r}, C \in \mathbb{C}^{s \times s}$, and $M=\left[\begin{array}{cc}A & B \\ 0 & C\end{array}\right]$, then there exists $M^{\#}$ if and only if there exist $A^{\#}, C^{\#}$, and $\left(I_{r}-A A^{\#}\right) B\left(I_{s}-C C^{\#}\right)=0$. Furthermore, when $M^{\#}$ exists, it is given by

$$
M^{\#}=\left[\begin{array}{cc}
A^{\#} & \left(A^{\#}\right)^{2} B\left(I_{s}-C C^{\#}\right)+\left(I_{r}-A A^{\#}\right) B\left(C^{\#}\right)^{2}-A^{\#} B C^{\#} \\
0 & C^{\#}
\end{array}\right] .
$$

From this theorem we have the following obvious consequence: Let $A \in \mathbb{C}^{r \times r}, B \in \mathbb{C}^{r \times s}$, and

$$
M=\left[\begin{array}{cc}
A & B \\
0 & 0
\end{array}\right]
$$

Then $M$ has group inverse if and only if $A$ is group invertible and

$$
\left(I_{r}-A A^{\#}\right) B=0 .
$$

Under this equivalence, one has

$$
M^{\#}=\left[\begin{array}{cc}
A^{\#} & \left(A^{\#}\right)^{2} B \\
0 & 0
\end{array}\right] .
$$

The following lemma, that extends the above consideration, will be needed in the sequel. 
Lemma 3.1. Let $\mathcal{A}$ be an algebra with unity, $q \in \mathcal{A}$ an idempotent, and $a \in \mathcal{A}$ such that $q a=a$. Then the following are equivalent

(i) $q a q \in \mathcal{A}^{\#}$ and $\left[1-a(q a q)^{\#}\right] a \bar{q}=0$.

(ii) $a \in \mathcal{A}^{\#}$.

Under this equivalence, one has

$$
a^{\#}=(q a q)^{\#}+\left((q a q)^{\#}\right)^{2} a \bar{q} .
$$

Note (previous to the proof). Since $q a=a$ we have $\bar{q} a q=\bar{q} a \bar{q}=0$, which means that in the representation (3.10) the two lower entries are zero; this generalizes when a $2 \times 2$ block matrix has null its lower blocks. The equality $\left[1-a(q a q)^{\#}\right] a \bar{q}=0$ generalizes the matrix relation (3.12). Moreover, the equality (3.14) clearly extends formula (3.13).

Proof. (i) $\Rightarrow$ (ii): Let $b=(q a q)^{\#}$. By definition of group inverse one has

$$
b q a q b=b, \quad q a q b q a q=q a q, \quad q a q b=b q a q .
$$

By Theorem 3.1, there exists $x \in \mathcal{A}$ such that $b=q x q$, and having in mind $q^{2}=q$, we get $b q=b=q b$, and thus, taking into account $q a=a$, relations (3.15) reduce to

$$
b a b=b, \quad a b a q=a q, \quad a b=b a q .
$$

Let $c=b+b^{2} a \bar{q}$. We will prove $a^{\#}=c$ by definition of the group inverse. First, we will simplify $a c$ :

$$
\begin{aligned}
a c= & a\left(b+b^{2} a \bar{q}\right)=a b+a b^{2} a \bar{q} \\
& =a b+(a b)(b a)-(a b)(b a q)=a b+(b a q)(b a)-(a b)(a b) \\
& =a b+b a(q b) a-a(b a b)=a b+b a b a-a b=b a .
\end{aligned}
$$

Now, we compute $c a$. Taking into account that $q a=a$, and thus $\bar{q} a=0$, we have

$$
c a=\left(b+b^{2} a \bar{q}\right) a=b a .
$$

These last two equalities show $a c=c a$. Next, we prove $c a c=c$ :

$$
c a c=(c a) c=(b a)\left(b+b^{2} a \bar{q}\right)=(b a b)(1+b a \bar{q})=b(1+b a \bar{q})=c .
$$

Finally, we prove $a c a=a$ : Until now, we have not used $(1-a b) a \bar{q}=0$. From this, and the middle relation of (3.16) we have $a b a=a$, and thus

$$
a c a=a(c a)=a(b a)=a b a=a .
$$

Since we have proved $a c a=a, c a c=b$, and $a c=c a$, we have gotten $a^{\#}=c$.

(ii) $\Rightarrow$ (i): Firstly, let us recall $q a^{\#}=q a^{\#} a a^{\#}=q a\left(a^{\#}\right)^{2}=a\left(a^{\#}\right)^{2}=a^{\#}$. Now, let us prove that $q a^{\#} q=(q a q)^{\#}$ by checking the three conditions of the group inverse. It is easy to see $(q a q)\left(q a^{\#} q\right)=a a^{\#} q=a^{\#} a q=\left(q a^{\#} q\right)(q a q)$. Moreover, one has

$$
(q a q)\left(q a^{\#} q\right)(q a q)=a a^{\#} a q=a q=q a q
$$

and similarly $\left(q a^{\#} q\right)(q a q)\left(q a^{\#} q\right)=q a^{\#} q$ holds. Finally, let us prove $\left[1-a(q a q)^{\#}\right] a \bar{q}=0$ :

$$
\left[1-a(q a q)^{\#}\right] a \bar{q}=\left(1-a q a^{\#} q\right) a \bar{q}=a \bar{q}-a q a^{\#} q a \bar{q}=a \bar{q}-a \bar{q}=0 .
$$

The lemma is proved. 
Theorem 3.3. Let $\mathcal{A}$ be an algebra with unity, $a \in \mathcal{A}$, and $p \in \mathcal{A}$ an idempotent. Assume that pap and $\bar{p} a \bar{p}$ have group inverse and

$$
p a \bar{p} a=0, \quad\left(1-p a(p a p)^{\#}\right) p a \bar{p}=0, \quad\left(1-\bar{p} a(\bar{p} a \bar{p})^{\#}\right) \bar{p} a p=0,
$$

then

$a^{\#}=(p a p)^{\#}+(\bar{p} a \bar{p})^{\#}+\left(1-(\bar{p} a \bar{p})^{\#} a\right)\left[(p a p)^{\#}\right]^{2} a \bar{p}+\left[(\bar{p} a \bar{p})^{\#}\right]^{2} a p\left(1-(p a p)^{\#} a\right)-(\bar{p} a \bar{p})^{\#} a(p a p)^{\#}$.

Proof. Let $a_{1}=p a$ and $a_{2}=\bar{p} a$. By hypothesis we have $a_{1} a_{2}=p a \bar{p} a=0$. We will use now Lemma 3.1 to prove that $a_{1}$ has group inverse. Since $p a_{1}=p(p a)=p a=a_{1}$, $p a_{1} p=p a p \in \mathcal{A}^{\#}$, and $\left(1-a_{1}\left(p a_{1} p\right)^{\#}\right) a_{1} \bar{p}=\left(1-p a(p a p)^{\#}\right) p a \bar{p}=0$ we have that $a_{1} \in \mathcal{A}^{\#}$ and

$$
a_{1}^{\#}=\left(p a_{1} p\right)^{\#}+\left(\left(p a_{1} p\right)^{\#}\right)^{2} a_{1} \bar{p}=(p a p)^{\#}+\left((p a p)^{\#}\right)^{2} p a \bar{p} .
$$

In a similar way, we use Lemma 3.1 to prove that $a_{2} \in \mathcal{A}^{\#}$; but, we will use the idempotent $\bar{p}$ instead of $p$ as before. In fact, since $\bar{p} a_{2}=a_{2}, \bar{p} a_{2} \bar{p}=\bar{p} a \bar{p} \in \mathcal{A}^{\#}$, and $\left(1-a_{2}\left(\bar{p} a_{2} \bar{p}\right)^{\#}\right) a_{2} p=$ $\left(1-\bar{p} a(\bar{p} a \bar{p})^{\#}\right) \bar{p} a p=0$, we get

$$
a_{2}^{\#}=(\bar{p} a \bar{p})^{\#}+\left((\bar{p} a \bar{p})^{\#}\right)^{2} \bar{p} a p .
$$

By Theorem 2.1 we have

$$
a^{\#}=\left(a_{1}+a_{2}\right)^{\#}=a_{2}^{\pi} a_{1}^{\#}+a_{2}^{\#} a_{1}^{\pi} .
$$

Next thing we do is simplifying $a_{1}^{\pi}$ and $a_{2}^{\pi}$. To this end, let us denote $b=(\text { pap })^{\#}$, and since $b=(p a p)^{\#} \in(p \mathcal{A} p)^{\#}$, by item (ii) of Theorem 3.1, we get $b p=b=p b$. Having in mind that $b=(p a p)^{\#}$ we get $p a p b=b p a p$, papbpap $=p a p$, and $b p a p b=b$ or equivalently,

$$
p a b=b a p, \quad \text { pabap }=p a p, \quad b a b=b .
$$

Now we have

$$
a_{1}^{\pi}=1-a_{1} a_{1}^{\#}=1-p a\left(b+b^{2} p a \bar{p}\right)=1-p a b-p a b^{2} p a \bar{p}=1-b a p-b a \bar{p}=1-b a .
$$

Similarly, if we denote $c=(\bar{p} a \bar{p})^{\#}$, we get $a_{2}^{\pi}=1-c a$. Therefore, recalling that $p b=b=b p$, $\bar{p} c=c=c \bar{p}$, and $c b=b c=0$, we obtain

$$
\begin{aligned}
a^{\#} & =a_{2}^{\pi} a_{1}^{\#}+a_{2}^{\#} a_{1}^{\pi} \\
& =(1-c a)\left(b+b^{2} p a \bar{p}\right)+\left(c+c^{2} \bar{p} a p\right)(1-b a) \\
& =b+b^{2} p a \bar{p}-c a b-c a b^{2} p a \bar{p}+c+c^{2} \bar{p} a p-c b a-c^{2} \bar{p} a p b a \\
& =b+b^{2} a \bar{p}-c a b-c a b^{2} a \bar{p}+c+c^{2} a p-c^{2} a b a \\
& =b+c+(1-c a) b^{2} a \bar{p}+c^{2} a p(1-b a)-c a b .
\end{aligned}
$$

This concludes the proof.

In what follows we shall apply Theorem 3.3 to the operator $M \in \mathcal{B}(X \times Y)$ represented

$$
M=\left[\begin{array}{ll}
A & B \\
C & D
\end{array}\right],
$$

where $X$ and $Y$ are Banach spaces, $A \in \mathcal{B}(X), B \in \mathcal{B}(Y, X), C \in \mathcal{B}(X, Y)$, and $D \in \mathcal{B}(Y)$. 
Theorem 3.4. Let $M$ be an operator of the form (3.17). If $A$ and $D$ are group invertible, $B C=0, B D=0, A^{\pi} B=0, D^{\pi} C=0$, then $M$ is group invertible and

$$
M^{\#}=\left[\begin{array}{cc}
A^{\#} & \left(A^{\#}\right)^{2} B \\
-D^{\#} C A^{\#}+\left(D^{\#}\right)^{2} C A^{\pi} & D^{\#}-D^{\#} C\left(A^{\#}\right)^{2} B-\left(D^{\#}\right)^{2} C A^{\#} B
\end{array}\right] .
$$

Proof. We will apply Theorem 3.3 identifying $M \leftrightarrow a$ and the idempotent

$$
P=\left[\begin{array}{cc}
I_{X} & 0 \\
0 & 0
\end{array}\right] \leftrightarrow p
$$

where $I_{Z}$ denotes the the identity operator in the Banach space $Z$. Since $A$ is group invertible in $\mathcal{B}(X)$ and $P M P=A \oplus 0$ we obtain that $P M P$ is group invertible in $\mathcal{B}(X \times Y)$ and $(P M P)^{\#}=A^{\#} \oplus 0$. In a similar way we get the group invertibility of $\bar{P} M \bar{P}$ and $(\bar{P} M \bar{P})^{\#}=$ $0 \oplus D^{\#}$. From $B C=0$ and $B D=0$ we easily get $P M \bar{P} M=0$. An evident computation shows

$$
\left[I_{X \times Y}-P M(P M P)^{\#}\right] P M \bar{P}=\left[\begin{array}{cc}
0 & A^{\pi} B \\
0 & 0
\end{array}\right] .
$$

Hence from the hypothesis of this theorem we get $\left[I_{X \times Y}-P M(P M P)^{\#}\right] P M \bar{P}=0$. Analogously, from $D^{\pi} C=0$ we obtain $\left[I_{X \times Y}-\bar{P} M(\bar{P} M \bar{P})^{\#}\right] \bar{P} M P=0$. We can apply Theorem 3.3 obtaining the formula of this theorem.

Theorem 3.5. Let $M$ be an operator of the form (3.17). If $A$ and $D$ are group invertible, $C A=0, C B=0, A^{\pi} B=0, D^{\pi} C=0$, then $M$ is group invertible and

$$
M^{\#}=\left[\begin{array}{cc}
A^{\#}-\left(A^{\#}\right)^{2} B D^{\#} C-A^{\#} B\left(D^{\#}\right)^{2} C & \left(A^{\#}\right)^{2} B D^{\pi}-A^{\#} B D^{\#} \\
\left(D^{\#}\right)^{2} C & D^{\#}
\end{array}\right] .
$$

Proof. This theorem has the same proof as the former Theorem 3.4, but now identifying $M \leftrightarrow a$ and the idempotent $P=0 \oplus I_{Y} \leftrightarrow p$,

For the following two results, we need to recall that given an operator $T \in \mathcal{B}(X, Y)$, the adjoint of $T$ (which generalizes the concept of the conjugate transpose of a matrix) is an operator $T^{*} \in \mathcal{B}\left(Y^{*}, X^{*}\right)$, where $Z^{*}$ denotes the dual space of the Banach space $Z$. It is easy to see that if $T \in X$ is group invertible, then $T^{*}$ is also group invertible and $\left(T^{\#}\right)^{*}=\left(T^{*}\right)^{\#}$. Also, as is easily seen from the definition, one has $\left(T^{*}\right)^{\pi}=\left(T^{\pi}\right)^{*}$.

Theorem 3.6. Let $M$ be an operator of the form (3.17). If $A$ and $D$ are group invertible, $B C=0, D C=0, C A^{\pi}=0, B D^{\pi}=0$, then $M$ is group invertible and

$$
M^{\#}=\left[\begin{array}{cc}
A^{\#} & A^{\#} B D^{\#}+A^{\pi} B\left(D^{\#}\right)^{2} \\
C\left(A^{\#}\right)^{2} & C\left(A^{\#}\right)^{2} B D^{\#}-C A^{\#} B\left(D^{\#}\right)^{2}+D^{\#}
\end{array}\right] .
$$

Proof. It is enough to apply Theorem 3.4 to operator $M^{*}$.

Theorem 3.7. Let $M$ be an operator of the form (3.17). If $A$ and $D$ are group invertible, $A B=0, C B=0, C A^{\pi}=0, B D^{\pi}=0$, then $M$ is group invertible and

$$
M^{\#}=\left[\begin{array}{cc}
A^{\#}-B D^{\#} C\left(A^{\#}\right)^{2}-B\left(D^{\#}\right)^{2} C A^{\#} & B\left(D^{\#}\right)^{2} \\
D^{\pi} C\left(A^{\#}\right)^{2}-D^{\#} C A^{\#} & D^{\#}
\end{array}\right] .
$$

Proof. As before, it is enough to apply Theorem 3.5 to operator $M^{*}$. 


\section{References}

[1] A. Ben-Israel, T. Greville. Generalized inverses. Theory and applications Springer, 2003.

[2] J. Benítez. Moore-Penrose inverses and commuting elements of $C^{*}$-algebras. J. Math. Anal. Appl. 345 (2008), 766-770.

[3] J. Benítez, Xiaoji Liu, Tongping Zhu. Nonsingularity and group invertibility of linear combinations of two $k$-potent matrices.

[4] C. Cao. Some result of group inverse of partitioned matrix on body. J. Nat. Sci. Heilongjiang Univ. 2001. 18(3), 5-7.

[5] N. Castro González, J.J. Koliha. New additive results for the $g$-Drazin inverse. Proc. Roy. Soc. Edinburgh Sect. A 134 (2004), 1085-1097.

[6] N. Castro González, E. Dopazo, M. F. Martínez Serrano. On the Drazin inverse of the sum of two operators and its application to operator matrices. J. Math. Anal. Appl. 350 (2009), 207-215.

[7] D.S. Cvetković-Ilić, D.S. Djordjević, Y. Wei. Additive results for the generalized Drazin inverse in a Banach algebra. Linear Algebra Appl. 418 (2006), 53-61.

[8] C.Y. Deng. The Drazin inverses of sum and difference of idempotents. Linear Algebra Appl. 430 (2009), 1282-1291.

[9] C. Deng, D. S. Cvetković-Ilić, Y. Wei. Some results on the generalized Drazin inverse of operator matrices. Linear Multilinear Algebra. 2009, (in press)

[10] D.S. Djordjević, Y. Wei. Additive results for the generalized Drazin inverse. J. Austral. Math. Soc. 73 (2002), 115-125.

[11] R.E. Hartwig, G.R.Wang, Y.Wei. Some additive results on Drazin inverse. Linear Algebra Appl. 322 (2001), 207-217.

[12] J. J. Koliha. The Drazin and Moore-Penrose inverse in $C^{*}$-algebras, Math. Proc. Roy. Irish Acad. 99 A (1999), 17-27.

[13] J. J. Koliha. Elements of $C^{*}$-algebras commuting with their Moore-Penrose inverse, Studia Math. 139 (2000), 81-90. 